\title{
«Y busqué su alma como a mi otro yo»: comentarios a Himerio 10.86-90*
}

\author{
Virginia Muñoz Llamosas \\ Universidad de Oviedo \\ munozvirginia@uniovi.es
«And I searched for his soul as my alter ego»: On Himerius 10.86-90

\begin{abstract}
En su discurso 10, un discurso propémptico de intencionada inspiración platónica, Himerio describe su reacción al conocer al alumno que ahora despide y afirma que, viéndose reflejado en él como en un espejo, buscó su alma como a su «otro yo». A partir de ahí analizamos las diferentes expresiones del griego para esta idea, que pasará a la tradición posterior bajo el latinismo alter ego, y que aparece fundamentalmente vinculada al tema de la amistad. Por su contenido el «otro yo» enlaza con el motivo literario del doble, que puede ser aprovechado negativa o positivamente (que es donde se situaría el amigo como "otro yo»). Teniendo en cuenta las diferentes expresiones para este concepto y las distintas reflexiones sobre la amistad, encontramos en este pasaje de Himerio, junto a la influencia de Platón, que él mismo pretendía, un eco, hasta ahora inadvertido, de Aristóteles y los proverbios acerca del amigo.
\end{abstract}

Palabras clave: Himerio; alter ego; otro yo; doble; amistad.

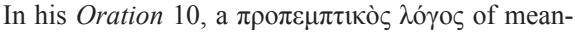
ingful platonic inspiration, Himerius describes his reaction on having known the pupil who now dismisses and affirms that, meeting reflected in him as in a mirror, he looked for his soul as to his «other myself». From there we analyze the different Greek expressions for this idea, which will go on to the later tradition under the Latinism alter ego, and that turns out to be fundamentally linked to the topic of friendship. The meaning of «other myself» connects with the literary motive of the double, which can be developed negatively or positively (where it would be placed the friend as «other myself»). Considering the different expressions for this concept and the different reflections on friendship, we find in this Himerius's passage, close to Plato's influence, which he himself was seeking, an echo, till now unnoticed, of Aristotle and the proverbs concerning friendship.

Key words: Himerius; alter ego; other self; double; friendship.

* Este trabajo forma parte del proyecto de investigación «La tradición literaria griega en los siglos III-IV d. C. Gramáticos, rétores y sofistas como fuentes de la literatura greco-latina» (Ref. [MICINN] FFI2011-30203-C02-01). 


\section{INTRODUCCIÓN}

De los setenta y un discursos conservados ${ }^{1}$ del rétor del siglo IV d. C. Himerio de Prusa $^{2}$, un número importante, diecisiete, son pronunciados con motivo de la llegada o despedida de uno o varios estudiantes o del propio

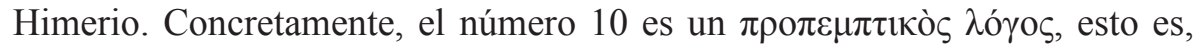
un discurso de despedida y buenos deseos para un alumno que se va. No nos ha llegado completo, sino que de él conservamos el extracto de Focio, fragmentos de los Excerpta Neapolitana ${ }^{3}$ y un comentario preliminar, $\pi \rho 0 \theta \varepsilon \omega \rho i ́ \alpha$, en el que se dan detalles importantes acerca de su composición. Este tipo de declamaciones era, a decir del propio Himerio ${ }^{4}$, reciente, y en ellos, según apunta Menandro el Rétor ${ }^{5}$ podemos encontrar consejos y recomendaciones para el que se va, especialmente si uno de mayor rango despide a otro infe-

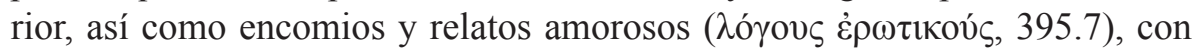

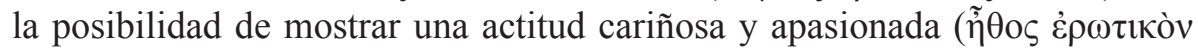

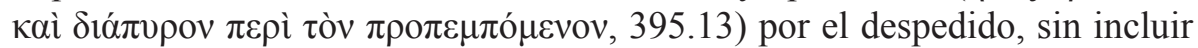
consejos cuando quien despide y el despedido tienen una dignidad similar, pero siempre con esos sentimientos que Menandro denomina $\dot{\varepsilon} \rho \omega \tau \imath \kappa \tilde{\omega} v \pi \alpha \theta \tilde{\omega} v$ (395.28). Himerio decide dar una apariencia más antigua al discurso que nos ocupa remontándose a Platón, de quien toma la forma de diálogo y el uso del mito para exponer ciertas ideas. Y, efectivamente, con un mito de indudables reminiscencias platónicas, recuerda Himerio que cuando Zeus creó la raza humana Eros no intervenía en los asuntos de los hombres, sino que únicamente disparaba sus flechas entre los dioses. Después, Zeus, preocupado por la posibilidad de que los hombres se extinguieran, hizo a Eros el guardián

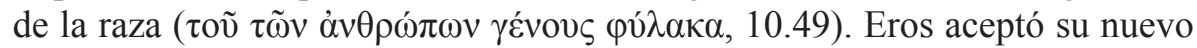
papel, pero consideró que no debía ocuparse de todas las almas, sino que

${ }^{1}$ De los setenta y uno que conoció Focio, tan sólo veinticuatro nos han llegado completos, de treinta y seis tenemos extractos, y de los once restantes, fragmentos.

${ }^{2}$ Una primera aproximación a este autor puede verse en Der Neue Pauly, 5, pp. 561-563, s. u. Himerios o en la introducción a la reciente traducción de Penella 2007.

3 Además de los manuscritos, en el caso de la transmisión textual de Himerio contamos con los extractos de Focio, los llamados Excerpta Neapolitana, que aparecen en un manuscrito del siglo XIV de Nápoles y los del Léxico de Andreas Lopadiota. Para el texto de Himerio seguiremos la edición de Colonna 1951. Para el resto de autores, de no indicarse lo contrario, seguimos las mismas ediciones que el $T L G$.

4 10.3-4.

${ }^{5}$ Men. Rh. 395. 
asignó el cuidado de las almas ordinarias a unos Eros vulgares ( $\pi \alpha v \delta \eta \dot{\mu o r} \varsigma$ ”Ep $\omega \sigma 1,10.53)$, hijos de las Ninfas, mientras que él se ocuparía de las almas

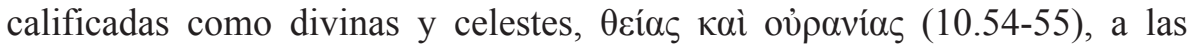

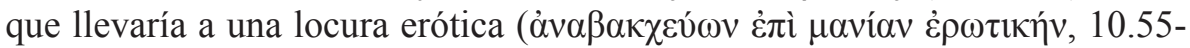
56). Sólo quienes han sido tocados por este Eros alcanzan con facilidad una

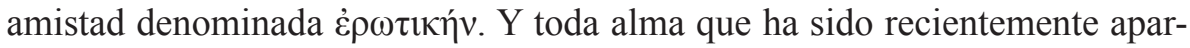
tada de la esfera superior y de la contemplación de la belleza, cuando la ve escondida en otra alma divina, desea unirse a ella, sintiendo el consuelo que le proporcionan el conocimiento y opinión verdaderos. Y eso es exactamente lo que Himerio sintió con este joven al que ahora despide, como vemos en sus palabras:

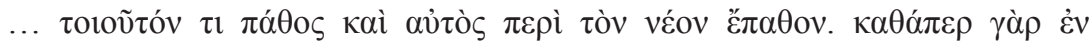

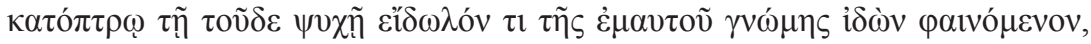

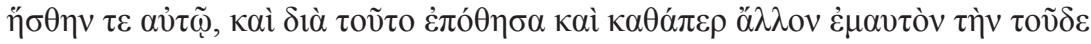

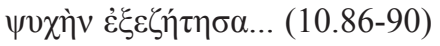

... un sentimiento semejante experimenté también yo acerca de este joven. Pues, viendo aparecer en su alma, como en un espejo, una imagen de mi propia manera de ser, me alegré y por esto deseé y busqué su alma como a mi otro yo.

La terminología empleada en todo el pasaje, así como la idea de que existen dos tipos de Eros, nos lleva inmediatamente a Platón y a las dos Afroditas ${ }^{6}$ de las que habla Pausanias en el Banquete ${ }^{7}$. Pero no es la influencia platóni$\mathrm{ca}^{8}$, sobre la que luego volveremos, el objeto de nuestro comentario, sino la expresión $\ddot{\alpha} \lambda \lambda$ ov $\dot{\varepsilon} \mu \alpha v \tau$ óv, «mi otro yo», cuya forma y contenido pasamos a analizar a continuación.

${ }^{6}$ También Himerio habla en 17.7 de dos Afroditas, una celeste y otra vulgar, que engendran a su vez muchos Eros celestes y vulgares; en cambio, en el pasaje que comentamos sólo existe un Eros celeste.

7 Pl., Smp. 180c ss.

${ }^{8}$ No es nuestro propósito tratar el tema de la amistad y Eros en Platón, para el que remitimos, por ejemplo, a Reeve 2011, con abundante bibliografía. 


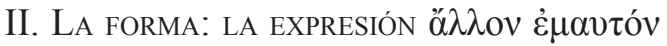

Estamos ante una expresión poco frecuente en griego, tan poco frecuente que sólo la utilizan, hasta donde sabemos por las búsquedas realizadas en el TLG, Himerio y dos autores del siglo XIV. El primero de ellos es Manuel Raúl ${ }^{9}$, que la usa en una carta en la que también se menciona esta idea en conexión con la más profunda amistad ${ }^{10}$, diciendo:

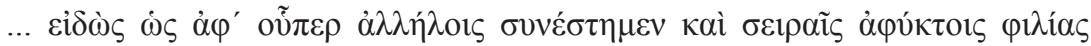

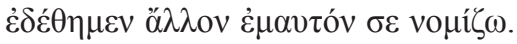

... sabiendo que, precisamente desde que nos unimos y fuimos atados por los lazos ineludibles de la amistad, yo te considero mi otro yo.

De forma semejante usa la expresión Constantino Acropolita ${ }^{11}$, de nuevo en una carta, que comienza con estas palabras:

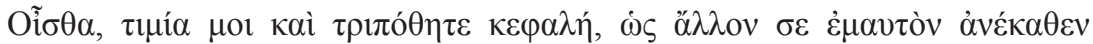

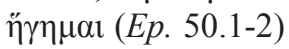

Sabes, mi querida y tres veces deseada cabeza, que desde el principio te he considerado mi otro yo.

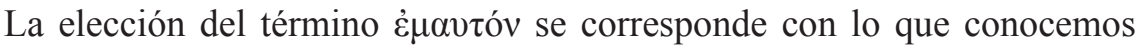
sobre la evolución de los pronombres reflexivos, y constituye una expresión muy precisa, que no deja dudas acerca del referente, que se identifica con el hablante, dado que los tres pasajes tienen los verbos en primera persona. Estas tres ocurrencias, que también coinciden en el caso acusativo, como se puede ver, están lejos del griego clásico. No obstante, y a pesar de lo tardío de su aparición, en la literatura anterior encontramos otras expresiones para

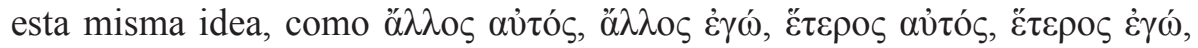

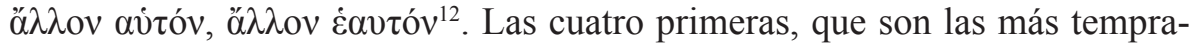
nas, aparecen mayoritariamente en nominativo, siempre en masculino y con

\footnotetext{
9 V. Fassoulakis 1973.

${ }^{10}$ Ep. 3.57.

11 Sobre este autor, v. Nicol 1965.

12 Rastreamos los precedentes de la expresión sobre todo allí donde se la utiliza hablando de la amistad, tema a propósito del cual la emplea Himerio. En cambio, pasaremos por alto
} 


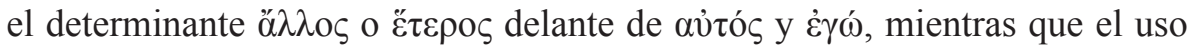
en otros casos (acusativo) es más tardío, como también es posterior la aparición de formas reflexivas.

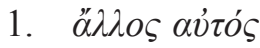

En el libro VIII de su Ética a Nicómaco ${ }^{13}$ Aristóteles trata el tema de la amistad y afirma que, en el caso de la más perfecta y equilibrada, el amigo es otro

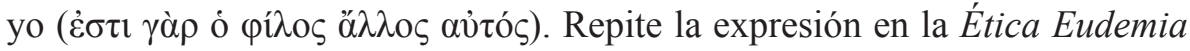
124529-30, donde dice:

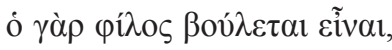

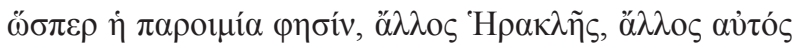

... el amigo quiere ser, como dice el proverbio, «otro Heracles», otro yo.

Este es un conocido proverbio, trasmitido, entre otros, por Plutarco ${ }^{14}$,

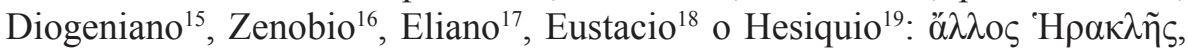
«otro Heracles», que se aplicaba a los atletas y a los forzudos en general ${ }^{20}$. En él se aprecia el uso de $\alpha \lambda \lambda \lambda$ o a una persona determinada, acepción ${ }^{21}$ que aparece recogida en el $D G E \mathrm{~s}$.u.

Volviendo a Aristóteles, sigue diciendo el filósofo que, sin embargo, existe una separación y es difícil que surja la unidad, y que a pesar de los posibles

su aparición en otros contextos, como, por ejemplo, en los autores cristianos, al tratar de la relación entre el Padre y el Hijo o las naturalezas de Cristo.

${ }^{13}$ EN $1166^{\mathrm{a} 33}$.

${ }^{14}$ Thes. 29.3.8.

${ }^{15} C P G$ I 1.63 .1$.

${ }^{16} C P G$ I 5.48 .1

17 VH 12.22.19.

${ }_{18}$ Commentarii ad Homeri Iliadem, p. 589, 41-45.

19 S. u. $\ddot{\alpha} \lambda \lambda$ os.

${ }^{20}$ Sobre el uso cómico y paródico que Teócrito hace de este proverbio, v. Montes Cala 2006.

${ }^{21}$ Este uso de $\alpha \ddot{\lambda} \lambda \mathrm{o}$ también tiene paralelos en latín en formas como alter y nouus. Por ejemplo, Cicerón se refiere a Metrodoro como paene alter Epicurus (fin. 2.92), y a Antonio como noue Hannibal (Phil. 13.25). En griego podemos ver cómo Epicteto (Diss. III, 22.76.3)

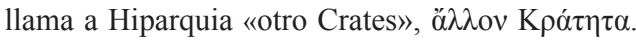


puntos en común entre los amigos, «un amigo desea existir como un yo se-

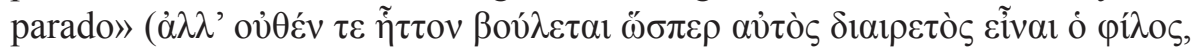
EE 1245³4-35).

En uno y otro pasaje podemos observar que $\alpha$ iós, que se define, según Adrados $^{22}$, como un pronombre nominal-adjetival, está funcionando como un sustantivo, y que es indiferente a las oposiciones personales: ya desde Homero en su uso nominal puede equivaler a cualquiera de las tres personas, aun-

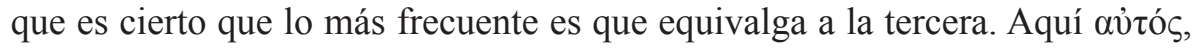
insistiendo en la identidad, en el ser mismo ${ }^{23}$ realizado en su plenitud de la persona o cosa a la que se refiere, alude a otro «él», a que el amigo es otro que se puede identificar plenamente con uno mismo, pero de la expresión griega no se deduce automáticamente la primera persona, por lo que la tra-

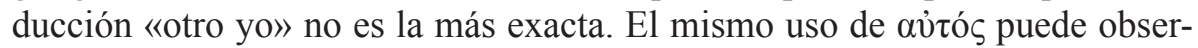
varse en la forma $\varphi$ í $\lambda \alpha v \tau o \zeta$, 'que se ama a sí mismo', o en otros compuestos donde $\alpha$ vós aparece como primer término: $\alpha$ $\tau$ ó $\beta o v \lambda o \varsigma$, 'que obra por propia

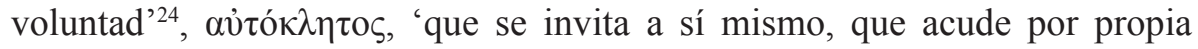

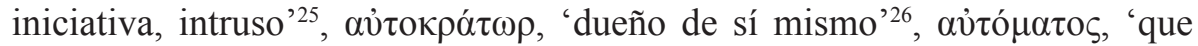
actúa por sí mismo'27. En todos ellos, $\alpha$ ${ }^{2}$ ó $\varsigma$ es claramente indiferente a la persona y, si bien estos compuestos, cuando aparecen en nominativo, suelen referirse a una tercera persona identificable con el sujeto gramatical, no faltan ejemplos con verbos en primera o segunda persona. Sí hace explícita la referencia a la primera persona la palabra «egoísta», con la que a menudo se traduce pí $\lambda \alpha v \tau o \varsigma$, una palabra que curiosamente llega a las lenguas romances muy tarde ${ }^{28}$, en el siglo XVIII, con un neologismo cuyo primer término es ego. De todos modos, hay que decir que en «egoísta» el pronombre personal ego del que deriva sufre un desplazamiento semántico, perdiendo su signifi-

22 Adrados 1992, p. 303.

23 Valor que, según Biraud 1987 y 1991, también aparece cuando se usa como determinante, y que engloba los valores de encarecimiento y exclusividad.

24 A., Th. 1054.

${ }^{25} \mathrm{Pl}$., Ep. (dub.) $331 \mathrm{~b} 6$.

${ }^{26}$ Th. 4. 63.

27 Ar., Pl. 1190.

${ }^{28}$ En castellano «egoísmo» se testimonia por primera vez en 1786; la forma latina de la que procede es un neologismo que aparece en 1722 (Pfaff 1722). En francés la primera mención es de 1755, y en italiano, de 1762. En francés, por otro lado, tenemos desde 1726 la forma égotiste. 
cado gramatical para adquirir un significado léxico, de manera que ya no se refiere al hablante y decimos «tú eres egoísta», «él es egoísta» y no *«tuísta» ni * «suísta» ${ }^{29}$. En cambio, otras lenguas modernas, como el inglés selfish o el alemán selbstsüchtig ${ }^{30}$ presentan una situación similar a la del griego, al utilizar un reflexivo como primer término del compuesto.

La forma aủ ós, por lo tanto, presenta aquí un uso nominal indiferente a la persona, con un valor enfático ${ }^{31}$ del que pueden derivar los sentidos de

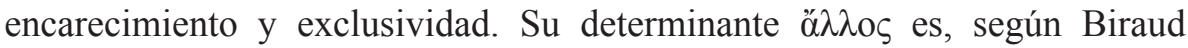
(1991), un adjetivo de alteridad que permite expresar la no identidad entre los objetos denotados por los nombres a los que determina $\mathrm{y}$, como hemos dicho anteriormente, puede designar 'un nuevo', 'un segundo' por oposición a una persona determinada.

Como paralelos latinos y, siguiendo, sin duda, a Aristóteles ${ }^{32}$, encontramos el testimonio de Cicerón, quien afirma en De amicitia 21.80: est [amicus] enim is quidem tamquam alter idem, con una expresión que podríamos traducir como «otro el mismo, un segundo él»y donde, como en el caso de

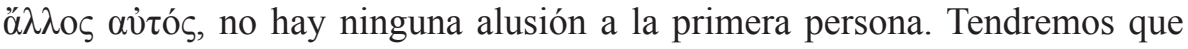
esperar a san Ambrosio, en el siglo IV d. C (De Spiritu sancto II 13.154, unde quidem interrogatus, quid amicus esset: alter, inquit, ego), para encontrar las palabras alter ego, que conservamos actualmente como latinismo ${ }^{33}$.

${ }^{29}$ Algo similar sucede con palabras como suidicio, donde la tercera persona puede ser primera o segunda.

${ }^{30}$ También tiene el alemán el cultismo egoistisch, al igual que el inglés tiene egoistical y egoist.

${ }_{31}$ Punto ya señalado por A. D., Pron. 2.1.1.

32 Rodríguez Donís 2009, pp. 147-167, afirma que las reflexiones de Cicerón sobre la amistad se inspiran en los peripatéticos, pero que no sabemos exactamente cuánto conoció de la obra de Aristóteles. En concreto, en p. 152 n. 26, pone en relación el ciceroniano alter

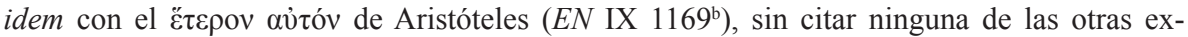
presiones que comentamos aquí, y limitándose a decir que «el origen de la fórmula es muy anterior a ambos».

${ }^{33}$ También usa san Ambrosio la expresión alteri tibi en referencia al amigo en De Off. III 133, añadiendo además el tópico de que los dos amigos pueden convertirse de dos seres en uno solo (unum ex duobus). Aparte de las fuentes clásicas que citamos aquí, Culbertson 1996, p. 153, ofrece pasajes bíblicos, de la patrística y autores árabes, donde también aparece la idea del amigo como otro yo. 


\section{2. $\ddot{\alpha} \lambda \lambda \sigma_{\circ} \dot{\varepsilon} \gamma \omega ́$}

Esta expresión aparece vinculada principalmente al tema de la amistad, e incluso existía un proverbio al respecto, que se remonta a la anécdota protagonizada por Pitágoras o, según otros testimonios, simplemente por los

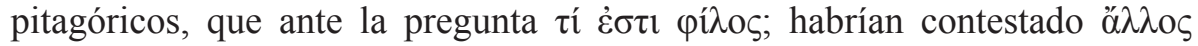
$\dot{\varepsilon} \gamma \omega^{34}$. También la usa Aristóteles inmediatamente después de haber usado la

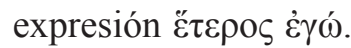

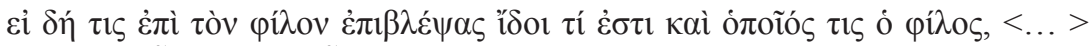

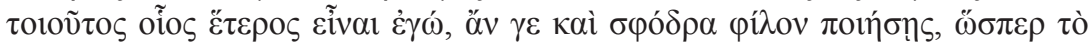

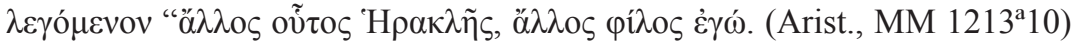

Ahora bien, si uno, mirando a su amigo viera qué es el amigo y cuál es su carácter y que es tal cual otro yo - al menos si imaginas un amigo muy íntimo-, según el dicho «este es otro Heracles», el amigo (será) otro yo.

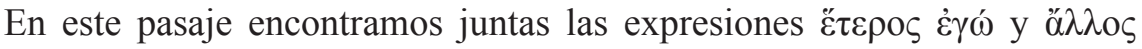
$\dot{\varepsilon} \gamma \omega ́$ aplicadas al amigo, con un uso de $\dot{\varepsilon} \gamma \omega \dot{~ e n ~ e l ~ q u e, ~ d e ~ m a n e r a ~ s i m i l a r ~ a ~ l o ~}$ que sucedía con la forma «egoísta», se pierde la referencialidad al hablante, por lo que, según el contexto, puede referirse a cualquier persona. Destaca, además, la alusión al proverbio antes comentado.

\section{3. $\check{\varepsilon} \tau \varepsilon \rho o \varsigma \alpha \dot{\tau o ́} \varsigma$}

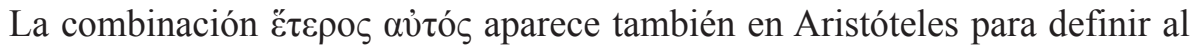

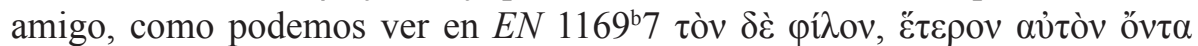

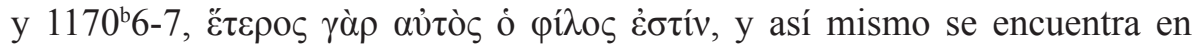

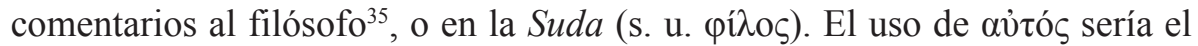
mismo que hemos explicado anteriormente, mientras que la forma ह̌ $\tau \varepsilon \rho \circ \varsigma$, a juzgar por el empleo que Aristóteles hace de ambos términos en pasajes muy

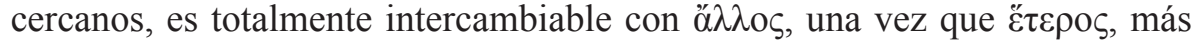

${ }^{34}$ Así lo transmiten Plu., Vit. Hom. 2.1904, Stob. II 33.13.2, Simp., in Epict. 87.25; cf. también Olymp., in Alc. 31.19, 36.8. Una ligera variante es proporcionada por la sentencia 296 recogida en el Gnomologium Vaticanum, que atribuye la respuesta a Zenón, y dice: Zńvஸv

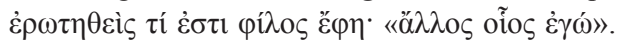

${ }^{35}$ Mich., in EN 516.31. 
marcado, pierde el sentido de 'otro de dos', exactamente igual que sucede con las formas latinas alter y alius.

\section{4. $\tilde{\varepsilon} \tau \varepsilon \rho o \varsigma \dot{\varepsilon} \gamma \dot{\omega}$}

De nuevo en Aristóteles y en conexión con el tema de la amistad encontramos

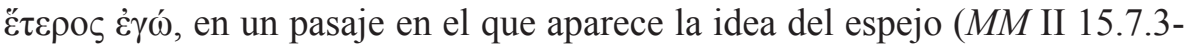
15.8.1) que posteriormente utilizará Himerio. Afirma el filósofo:

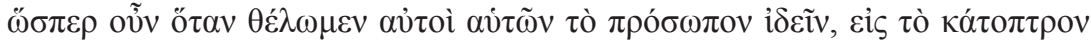

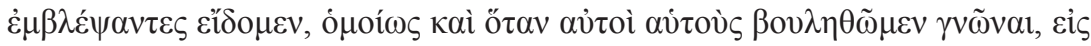

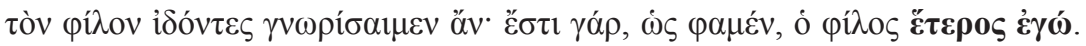

... Así pues, igual que cuando queremos ver nuestro propio rostro, lo vemos mirando al espejo, del mismo modo también cuando queramos conocernos a nosotros mismos, podríamos llegar a conocernos mirando al amigo; pues el amigo es, según decimos, otro yo.

Claramente se deduce de este pasaje que se llega a la propia identidad a través de lo otro, de la percepción del otro, que en el caso del amigo es identificable con uno mismo, mientras que la contemplación directa de uno mismo es, según el filósofo, imposible ${ }^{36}$.

Clemente de Alejandría se aparta ligeramente de Aristóteles al dar la de-

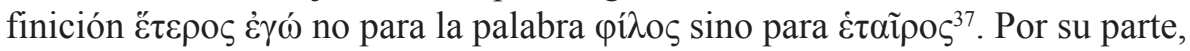
Jámblico $^{38}$ recupera la anécdota ya comentada sobre Pitágoras con esta modificación, al igual que Filón ${ }^{39}$, que con anterioridad hacía referencia al pre-

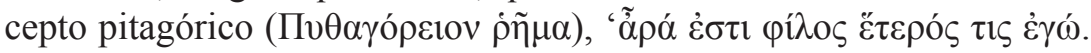

${ }^{36}$ MM II 15.6.1-15.7.3. Quizá ello se deba a que, como sostiene Laín Entralgo 1983, los griegos no llegaron a sentir la peculiar realidad del otro «yo», sólo conocen el «yo» visto desde fuera, por los demás, y ni Platón ni Aristóteles conocieron lo que hoy se llama el problema del otro, porque, sencillamente, no reflexionaron sobre lo que los filósofos postcartesianos llaman el yo personal, sino sobre el alma, lo que para el hombre moderno es la cosificación del yo.

${ }^{37} \mathrm{Clem}$. Al., Strom. II 9.41.2.4.

${ }^{38}$ Iambl., In Nic. 35.6.

${ }^{39}$ Fr., Gen. $17 b$. 


\title{
5. $\ddot{\alpha} \lambda \lambda o v \alpha \dot{\tau o ́ v}$
}

Tras las cuatro primeras variantes, todas ellas testimoniadas en Aristóteles y con mayor o menor continuidad en la literatura posterior, encontramos un nuevo testimonio en Plutarco ${ }^{40}$, que presenta la novedad de incorporar el reflexivo bajo la forma aviós, probablemente debido a la evolución de aう̉ hacia mero pronombre de tercera persona.

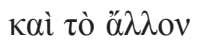

$\alpha$

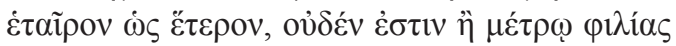

$\tau \tilde{n} \delta v \alpha ́ \delta 1 \chi \rho \omega \mu \varepsilon ́ v \omega v$.
\end{abstract}

... y el considerar al amigo «otro él mismo» y llamarle «compañero» en el sentido de 'el otro' no es sino servirse de la dualidad como medida de la amistad.

\section{6. $\ddot{\alpha} \lambda \lambda o v \dot{\varepsilon} \alpha v \tau o ́ v$}

Porfirio $^{41}$, en la Vida de Pitágoras afirma que este filósofo, a quien se le

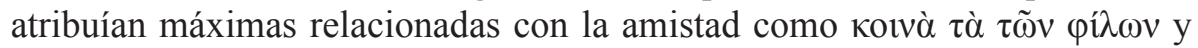

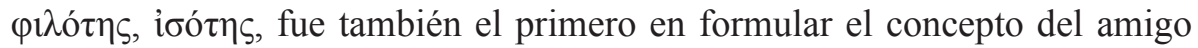
como alter ego.

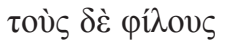

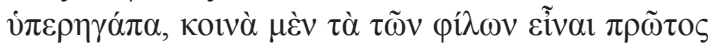

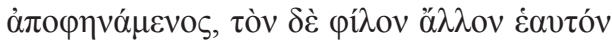

Y quiso a los amigos sobremanera, y fue el primero que mostró que son comunes las cosas de los amigos y que el amigo es «otro él».

Al margen de si realmente fue o no Pitágoras quien definió al amigo como «otro él mismo», lo que nos interesa es la nueva variante en la expresión, que está en consonancia con lo que vamos a encontrar a continuación. Al testimonio de Porfirio se suma el de $\operatorname{Sinesio}^{42}$, que sigue la línea iniciada por Plutarco con el uso del reflexivo, recordando la anécdota sobre Pitágoras;

\footnotetext{
${ }^{40}$ Plu. 2.93e9.

41 VP 33.1-3.

42 Ep. 100.16-17.
} 


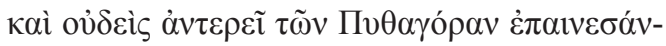

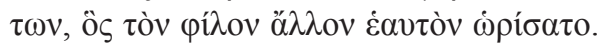

Y nadie se opondrá a los que alabaron a Pitágoras, que definió al amigo como «otro él».

Muy similar es el testimonio de su contemporáneo Basilio de Cesarea $^{43}$ :

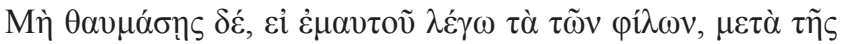

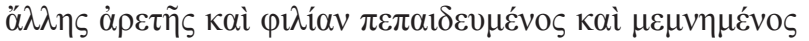

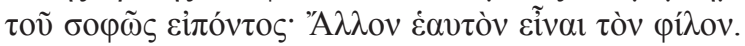

Y no te asombres si llamo «propias» las cosas de mis amigos, pues junto a las otras virtudes también he sido instruido en la amistad, y recuerdo al sabio diciendo: «el amigo es otro él mismo».

Y, ya en el siglo XIV, reencontramos la expresión en Manuel Calecas ${ }^{44}$, que en una de sus cartas dice:

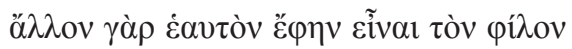

... pues decía que el amigo es otro él mismo.

Todos los testimonios son, como puede verse, muy similares: incorporan el reflexivo sin determinación expresa de la persona a la que se refieren, y tienen en común el caso acusativo.

Por otro lado, remontándonos al comienzo de la literatura griega, podemos hallar un precedente de estas expresiones en Homero. Como es sabido, en ese momento no existe un concepto unitario del «yo», sino que el hombre es entendido como la suma de distintas partes que lo integran ${ }^{45}$, y la referencia a la totalidad de la persona a través de la mención de una de sus partes es una

${ }^{43}$ Ep. 83.1.17-19.

${ }^{44}$ Sobre este autor, v. Kazhdan 1991 s. u. Kalekas.

${ }^{45}$ Se trata de una creencia común entre los pueblos primitivos, para quienes la imagen no es una reproducción del original distinta de este, sino el mismo original; de ahí el temor a la manipulación de la sombra/imagen/reflejo/retrato. En la literatura fantástica aparecen frecuentemente sombras o imágenes reflejadas que cobran vida y se rebelan, Esto nos habla de un pavor a la fragmentación, al mismo tiempo que demuestra que los temores primitivos no son ajenos al hombre moderno. 
metonimia muy común ${ }^{46}$. En el canto 18 de la Ilíada (Il. XVIII 80-82) Aquiles le dice a su madre que la delicada situación que atraviesan los aqueos, que él mismo había deseado, no le importa ya, una vez muerto Patroclo a manos de Héctor:

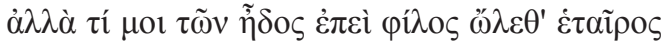
Пát ĩ $\sigma \circ v \dot{\varepsilon} \mu \tilde{n} \kappa \varepsilon \varphi \alpha \lambda \tilde{n}$;

Mas, ¿qué placer obtengo yo de esto, después de que murió mi compañero Patroclo, al que estimaba por encima de todos los demás, igual que a mi propia cabeza?

Las palabras ĩ $\sigma o v \dot{\varepsilon} \mu \tilde{\eta} \kappa \varepsilon \varphi \alpha \lambda \tilde{\eta}$, pueden interpretarse de dos formas. La primera, tal como hace, por ejemplo, López Eire ${ }^{47}$, establece una comparación entre el modo en que Aquiles estima a Patroclo y estima a su cabeza (esto es, a sí mismo). Pero también podríamos entender que ĩoov se refiere a la forma $\tau o ́ v$, cuyo antecedente es Patroclo, con lo que Aquiles estaría diciendo que su amigo es 'igual a mi cabeza', identificándose con él de manera semejante a como veremos después en la anécdota protagonizada por los pitagóricos, que precisamente mencionan tanto los escolios como Eustacio ${ }^{48}$ en sus comentarios a estos versos. Así lo entiende también Pizzolato ${ }^{49}$, para quien hay entre ambos héroes unos lazos que configuran ya una relación de recíproca pertenencia (de alter ego, dice concretamente) que se refleja en

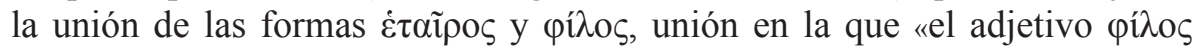

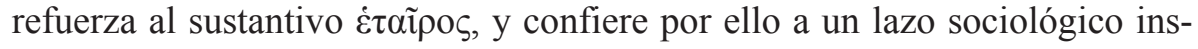

${ }^{46}$ Cf. por ejemplo, Il. XVII 242 o XVIII 114. Pueden verse otros usos similares de $\kappa \varepsilon \varphi \alpha \lambda \eta ́$ en Onians 1989, p. 97 ss. La metonimia sigue viva, más de dos mil años después, en las palabras de Constantino Acropolita que hemos comentado supra.

47 López Eire 1989.

${ }^{48}$ En el escolio a estos versos (Scholia in Homerum, Scholia in Iliadem [scholia uetera]) se recuerda precisamente la anécdota protagonizada por los pitagóricos respecto a la definición de amigo, y también Eustacio (Commentarii ad Homeri Iliadem p. 1131, 56; p. 1225, 27)

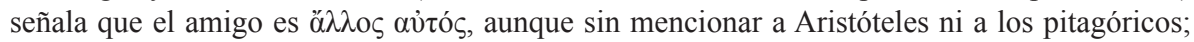
sí que alude al proverbio en Commentarii ad Homeri Iliadem p. 1359, 61 y también en Commentarii ad Homeri Odysseam, p. 1812, 53-54, refiriéndose a la amistad entre Odiseo y Néstor.

${ }^{49}$ Pizzolato 1996, p. 32. 
titucionalizado una carga de amor que personaliza el pacto genérico de la Kriegskameradschaft, 'camaradería guerrera'.

Se observa, por lo tanto, que en griego hay diferentes expresiones, más o menos marcadas, para la idea de «otro él»//«otro yo». Dada la indiferencia-

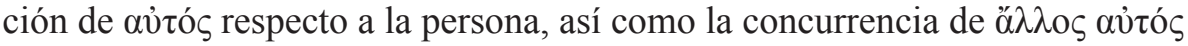

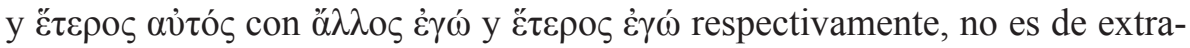

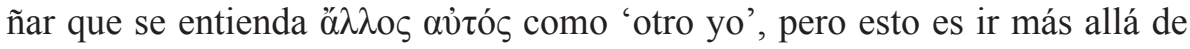
lo que los propios textos dicen. Entre todos los testimonios recogidos pueden hacerse claramente dos grupos: por un lado, aquellos en que aparecen av่tó $\varsigma$ o $̇ \gamma \omega ́$ y, por otro, aquellos en que aparece el reflexivo. En este segundo grupo tenemos, a su vez, dos posibilidades, los que mantienen la indiferenciación sobre la persona (aunque en la práctica sea una tercera) y los que marcan positivamente la primera, quizás arrastrados por aparecer con verbos también en primera persona, si bien destaca el hecho de que esa primera persona se ha desgramaticalizado y ya no coincide con el hablante. El hecho de que desde la koiné aủ ós se convierta en un simple pronombre de tercera persona no correferencial con el sujeto hace necesario el empleo del reflexivo, que puede aparecer de una forma más o menos marcada: en el caso concreto de

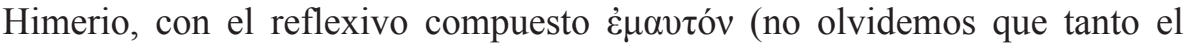
pasaje de Himerio como los dos posteriores tienen el verbo en primera persona), que prácticamente elimina el uso reflexivo que en origen podían tener tanto los pronombres personales como av̉ tóv.

\section{El CONTENIDO: LA IDEA DE OTRO YO}

En todos estos pasajes se ha tratado de la idea de «otro él»/《otro yo», de un alter ego, expresión que, según Herrero Llorente ${ }^{50}$, significa 'persona de confianza que representa a otra, amigo íntimo'. En psicología, alter ego suele entenderse como la segunda personalidad de alguien, que puede dar lugar a distintas alteraciones y patologías, mientras que en literatura hablar de un alter ego, de «otro yo», nos lleva al motivo del doble ${ }^{51}$, también conocido como «sosias», «el otro», «mi segundo yo» o Doppelgänger. El tema del

${ }^{50}$ V. Herrero Llorente 1980, s. u.

${ }^{51}$ Sobre las denominaciones «tema», «motivo», etc., v. la introducción a la tesis doctoral de Martín López 2006. Un estudio clásico sobre el tema del doble, el de Rank 1976, destaca especialmente el mito de Narciso, pero en él no hay interacción entre la imagen de Narciso, 
doble conoce un gran desarrollo a partir del Romanticismo, momento en el que las interpretaciones psicoanalíticas verán en el doble una materialización del lado oscuro del hombre, de su sombra, según la terminología junguiana. Pero, lejos de esa conexión con el lado oscuro tan propia de la época, encontramos el tema ya en la Antigüedad, en relación con dos personajes iguales cuya aparente identidad puede dar lugar a enredos y equívocos, razón por la que es explotado sobre todo en la comedia, como sucede, por ejemplo, en el Anfitrión de Plauto ${ }^{52}$, donde Júpiter suplanta al marido de Alcmena y Mercurio, a su criado Sosias, o en los Menaechmi, donde dos gemelos, que han vivido separados durante mucho tiempo, se reencuentran. En el primer caso, la usurpación de la identidad de otro individuo es consciente y deliberada, pues Júpiter y Mercurio suplantan a Anfitrión y Sosias para que Júpiter pueda tener a Alcmena. En cambio, en el segundo caso el engaño es involuntario, y los gemelos se reencuentran después de que uno de ellos hubiera desaparecido siendo niño. Precisamente el tema de los gemelos es considerado como una de las raíces antropológicas del motivo del doble ${ }^{53}$, junto al simbolismo en torno al alma y sus posibles manifestaciones en la sombra, el reflejo, el retrato o la estatua, así como la creencia de que, en determinadas circunstancias, el alma pueda abandonar el cuerpo.

En un estudio que aplica los principios del estructuralismo, Doleze ${ }^{54}$ habla de tres variantes posibles de este motivo: el llamado tema de Anfitrión, donde dos individuos homomorfos coexisten en un único mundo ficticio y presentan similitudes físicas o de comportamiento, que dan lugar a equívoco; en segundo lugar, el tema de Orlando, donde un solo individuo existe en mundos ficticios alternativos; y, por último, el tema del doble propiamente dicho, donde encontramos dos encarnaciones diferentes, habitualmente antagonistas, de un único individuo en un solo mundo ficticio, con las posibilidades de que los dobles sean simultáneos o exclusivos, y de que haya o no autentificación. La idea de otro yo en relación con la amistad, que no es contemplada por este autor, estaría próxima al tema de Anfitrión, en el sentido de que hay dos individuos diferentes que coexisten en un mismo mundo, pero no hay tensión ni

su «doble», y los demás: la imagen tan sólo es «otro» para él. Para una aproximación general, v. Fernández Bravo 1996.

52 V. García Hernández 2001.

53 V. Martín López 2006, p. 77 ss.

${ }^{54}$ Dolezel 1985. 
se vive en este caso el doble como algo ajeno y problemático. Ciertamente, la percepción de un desdoblamiento, de la existencia de un individuo cuya identidad parece repetir la propia, no provoca ninguna angustia cuando el otro yo es el amigo. Aquí el doble es complementario y no rival, y estamos lejos del aprovechamiento, que podríamos calificar de negativo ${ }^{55}$, del motivo literario del doble hacia la parte oscura, la animalidad e irracionalidad que subyace dentro de nosotros y acecha y fascina a la conciencia ${ }^{56}$. Esto es algo ajeno a las literaturas griega y latina ${ }^{57}$, donde los escasos usos del doble sirven para suplantar la personalidad, con una explotación cómica del motivo en el Anfitrión de Plauto, o con una vertiente trágica y que nos lleva a la desesperanza más absoluta en el caso del $\varepsilon$ í́ $\omega \lambda$ ov de Helena en la tragedia de Eurípides.

Muy al contrario, estaríamos ante otra posibilidad totalmente distinta, la que se orienta hacia el «yo ideal», el «alma gemela», la identidad soñada de la plena armonía con el cosmos o de la identificación y fusión de dos individuos originariamente independientes en uno solo, en lugar de la escisión de un solo yo en dos. Resulta, por lo menos, curioso, el hecho de que el tema del doble, del alter ego, no se haya puesto en relación con el tema del amigo, que es el que, como hemos podido ver, da lugar en la literatura griega a las expresiones que hemos repasado anteriormente. Lo que sucede es que el doble, que en principio era el yo idéntico, se ha convertido en el yo opuesto, perdiendo así toda posible vinculación con el amigo.

La amistad más perfecta que se puede concebir sería aquella en la que los dos amigos parecen en realidad un solo hombre, y hay la mayor semejanza posible entre ellos, lo que genera las expresiones antes comentadas, y también otras de uso común que son recogidas por Aristóteles ${ }^{58}$, como $\mu$ íav

55 En la literatura clásica no encontramos apenas ejemplos del tema del doble en su vertiente negativa, si exceptuamos el caso de Helena y su عí $\delta \omega \lambda o v$, cuyo análisis excede el propósito de este trabajo, y que curiosamente no es mencionado por los autores que hacen un estudio estructural del tema, mientras que en la abundante bibliografía que existe sobre el mito de Helena, sus versiones y las obras de Estesícoro y Eurípides, no se profundiza en la relación entre esta versión del mito y el motivo literario del doble.

${ }^{56}$ Son palabras de Herrero Cecilia 2006, p. 68.

${ }^{57}$ Pero tal vez no sea ajeno al pensamiento griego, concretamente a las ideas que Platón expone en el Fedro sobre la existencia de una duplicidad de las cosas, un lado siniestro percibido como negativo, v. Ávila Crespo 2000.

${ }^{58}$ EN 9.8.1168 , aunque en realidad Aristóteles no reflexiona sobre ello en este pasaje, pues lo que está cuestionando es si uno debe amarse ante todo a sí mismo, y si es o no vergonzoso el ser egoísta. Plutarco nos habla también de ello en Moralia 96 d-f. 


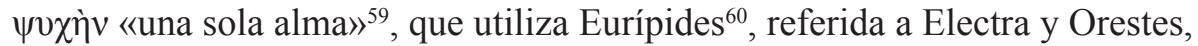
y que tanto recuerda al animae dimidium meae con que Horacio se dirige a Virgilio $^{61}$. Pero hablar de una sola alma no es exactamente lo mismo que afirmar la existencia de dos almas independientes que se identifican entre sí: lo primero podría habernos llevado a una unión con el amigo similar a la que propone Platón en el Banquete con el andrógino y la añorada unidad perdida, que es deseada por cada $\operatorname{mitad}^{62}$, de igual modo que en Himerio su alma desea el alma del amigo. Sin embargo, Himerio no utiliza la idea del andrógino - aunque sí repite palabras como el verbo $\pi$ o $\theta \varepsilon ́ \omega$ o el compuesto

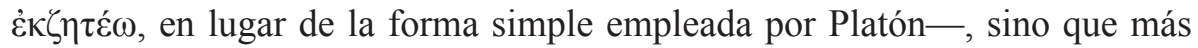
bien parece inspirarse en otro pasaje platónico, concretamente del Fedro, donde Sócrates también habla acerca del amor. Tras afirmar que toda alma es inmortal y que sólo las que han visto la verdad pueden tomar forma humana (249b 5-6) Sócrates le explica a Fedro una cuarta forma de locura ( $\mu \alpha v i ́ \alpha)$ que se produce cuando alguien contempla la belleza de este mundo y, recordando la verdadera, le salen alas y quiere volar, pero como no puede, mira hacia arriba y se olvida de las cosas de aquí, por lo que se lo toma por loco. Recordar los seres verdaderos que hemos contemplado no es algo fácil, pero puede conseguirse a través de la visión de la belleza, cuyos efectos son descritos en un largo pasaje que comienza de la siguiente manera:

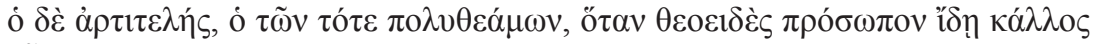

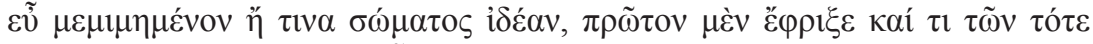

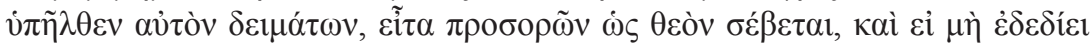

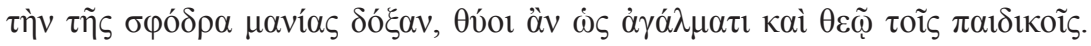
(251a 1-7)

... aquel cuya iniciación es todavía reciente, el que contempló mucho de las de entonces, cuando ve un rostro de forma divina, o entrevé, en el cuerpo, una idea que imita bien a la belleza, se estremece primero, y le sobreviene algo de los temores de antaño y, después, lo venera, al mirarlo, como a un dios, y si no

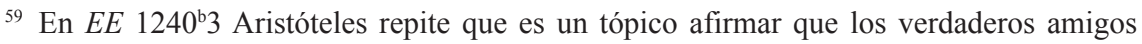

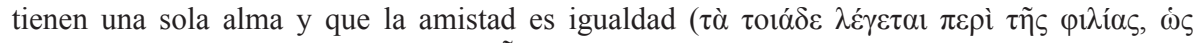

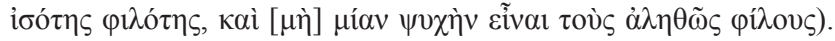

${ }^{60}$ Or. 1045.

${ }^{61}$ C. I 3.8.

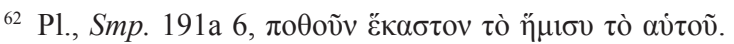


tuviera miedo de parecer muy enloquecido, ofrecería a su amado sacrificios como si fuera la imagen de un dios.

Este es el efecto que el amado tiene sobre el amante, una pasión a la que los hombres llaman amor y en la que los amantes tratan de llevar al amado a una total semejanza con ellos mismos y con los dioses que veneran (Eis

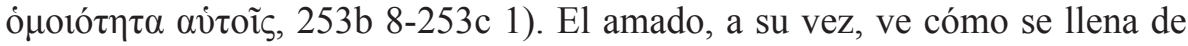
amor su alma, aunque, en palabras de Sócrates, «no acierta a qué atribuirlo y se olvida de que, como en un espejo, se está mirando a sí mismo en el aman-

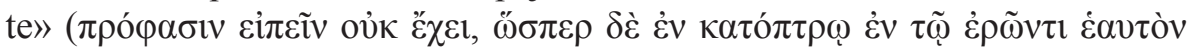
ó $\tilde{v} v \lambda \varepsilon \dot{\varepsilon} \lambda \eta \varepsilon \varepsilon v, 255 \mathrm{~d}$ 5-6), y siente algo que Sócrates define como anti-amor, como un reflejo del amor, aunque está convencido de que es amistad y así la llama. Hay que recordar que para Platón la diferencia entre amistad y amor, entre el amigo y el amado, es una cuestión de intensidad, mientras que Aristóteles sí hace una separación entre ambos sentimientos y reflexiona sobre la amistad sin ponerla en relación con el amor ${ }^{63}$, y entendiéndola no como un

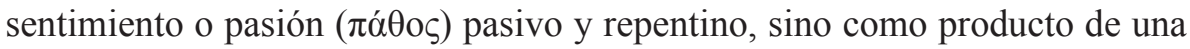
elección ( $(\xi \xi 1 \varsigma)$ activa y consciente ${ }^{64}$.

\section{Conclusión}

Para expresar sus sentimientos hacia el discípulo que se va, recurre Himerio, por lo tanto, a la idea platónica de un alma que ve en otra (la del amado en Platón o la del amigo en Himerio) una belleza que le hace recordar la visión de la verdadera realidad que un día tuvo. Y, muy probablemente, también está tomada de Platón la comparación con la contemplación en el espejo ${ }^{65}$, que hemos reencontrado en Aristóteles. Himerio ha cumplido lo que anunciaba en la $\pi \rho 0 \theta \varepsilon \omega \rho i ́ \alpha$ del discurso remontándose a Platón, no sólo en la forma dialogada (que apenas apreciamos, dado el estado fragmentario del discurso), sino

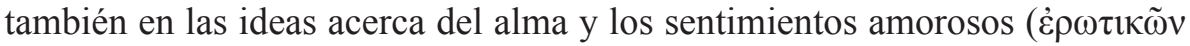

${ }^{63}$ V. un tratamiento más amplio del concepto de amistad en ambos filósofos en Pizzolato 1996, pp. 66-106, Vallejo Campos 2009, Vallejo Campos 2002, Calvo Martínez 2002 o Smith Pangle 2008, especialmente el capítulo «Friends as other selves».

${ }^{64}$ V. Pizzolato 1996, pp. 83-84 y, de forma muy similar, Vallejo Campos 2009, sobre la

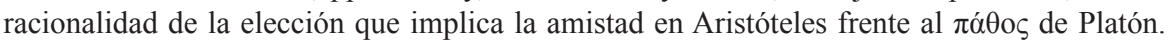

${ }^{65}$ Himerio retoma el tema del espejo en 48.12 , donde repite la idea de que las almas divinas y recién iniciadas tienden a lo mejor. 
$\pi \alpha \theta \tilde{\omega} v)$ que Menandro el Rétor veía como típicos de los discursos de despedida. A esta deseada y expresa inspiración platónica se suma, en nuestra opinión, un eco, hasta ahora inadvertido, bien de Aristóteles, bien de los tradicionales proverbios acerca del amigo, que nos llega a través de las palabras $\ddot{\alpha} \lambda \lambda$ ov $\dot{\varepsilon} \mu \alpha v \tau o ́ v$, que forman parte de una serie de expresiones, muy similares entre sí, que encontramos en griego para referirnos al concepto de «otro yo». Estas fórmulas aparecen, como hemos visto, fundamentalmente en relación con el tema de la amistad y son origen del conocido latinismo alter ego.

\section{BiBLIOGRAFÍA}

Adrados, F. R. 1992: Nueva sintaxis del griego antiguo, Madrid.

Ávila Crespo, R. 2000: «Identidad y alteridad. Una aproximación filosófica al problema del doble», $\Delta \alpha i ́ \mu \omega v$, Revista de Filosofia 20, pp. 5-23.

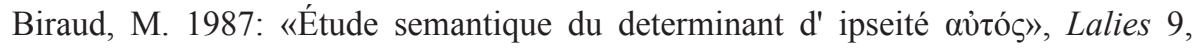
pp. $95-98$.

Biraud, M. 1991: La determination du nom en grec classique, Niza.

Calvo Martínez, T. 2002: «La concepción aristotélica de la amistad», IX Jornadas sobre la amistad. La amistad en la antigua Grecia, <http://antiqua.gipuzkoakultura.net/amistad_eu.php> (09/09/2012).

Colonna, A. 1951: Himerii. Declamationes et orationes cum deperditarum fragmentis, Roma.

Culbertson, P. L. 1996: «Men and Christian friendship», en Krondorfer, B. (ed.), Men's bodies, men's gods, Nueva York, pp. 149-180.

Dolezel, L. 1985: «Le triangle du double; un champ thématique», Revue Poétique 64, pp. 463-472.

Fassoulakis, S. 1973: The Byzantine family of Raoul-Ral(l)es, Atenas.

Fernández Bravo, N. 1996: «Doubles and Counterparts», en Brunel, P. (ed.), Companion to literary myths, heroes and archetypes, Londres, pp. 342-382.

García Hernández, B. 2001: Gemelos y sosias. La comedia del doble en Plauto, Shakespeare y Molière, Madrid.

Herrero Cecilia, J. 2006: «El mito como intertexto: la reescritura de los mitos en las obras literarias», Çedille. Revista de estudios franceses 2, pp. 58-76.

Herrero Llorente, V. J. 1980: Diccionario de expresiones y frases latinas, Madrid.

Kazhdan, A. P. (ed.) 1991: The Oxford Dictionary of Byzantium, Nueva York.

Laín Entralgo, P. 1983: Teoría y realidad del otro, Madrid.

López Eire, A. 1989: Homero. Ilíada, Madrid.

Martín López, R. 2006: Las manifestaciones del doble en la narrativa breve española contemporánea, Universidad Autónoma de Barcelona. 
Montes Cala, J. G. 2006: «Heracles en el Idilio IV de Teócrito», en Calderón, E., Morales, A. y Valverde, M. (eds.), Koinós lógos. Homenaje al profesor José García López, Murcia, pp. 657-668.

Nicol, D. M. 1965: «Constantine Akropolites. A prosopographical note», Dumbarton Oaks Papers 19, pp. 249-256.

Onians, R. B. 1989: The origins of European thought, Cambridge.

Penella, R. J. 2007: Man and the Word. The orations of Himerius, Berkeley-Los Ángeles.

Pfaff, C. M. 1722: Oratio de egoismo, nova philosophia haeresi, Tubinga.

Pizzolato, L. 1996: La idea de la amistad en la Antigüedad clásica y cristiana, Barcelona.

Rank, O. 1976: El doble, Buenos Aires.

Reeve, C. D. C. 2011: «Plato on friendship and Eros», en Zalta, E. N. (ed.), The Stanford Encyclopedia of Philosophy (Spring 2011 Edition), <http://plato.stanford.edu/> (09/09/2011).

Rodríguez Donís, M. 2009: «La amistad en Cicerón: crítica del utilitarismo», en Zamora Calvo, J. M. (ed.), La amistad en la filosofía antigua, Madrid, pp. 147-167.

Smith Pangle L., 2008: Aristotle and the philosophy of friendship, Cambridge-Nueva York.

Vallejo Campos, A. P. 2002: «Elogio de la locura: amistad y amor en Platón y Aristóteles», en IX Jornadas sobre la amistad. La amistad en la antigua Grecia, $<$ http://antiqua.gipuzkoakultura.net/amistad_eu.php> (09/09/2012)

Vallejo Campos, A. P. 2009: «Éros y Philía en Platón y Aristóteles», en Zamora Calvo, J. M. (ed.), La amistad en la filosofía antigua, Madrid, pp. 63-78.

Fecha de recepción de la primera versión del artículo: 11/07/2011 Fecha de aceptación: 06/09/2012

Fecha de recepción de la versión definitiva: 14/09/2012 